\title{
Perturbative expansion using variational phonon basis for Holstein model
}

\author{
Jayita Chatterjee ${ }^{1}$, A. N. Das ${ }^{1}$ and P. Choudhury ${ }^{2}$ \\ 1 Saha Institute of Nuclear Physics \\ 1/AF Bidhannagar, Calcutta 700 064, India \\ 2 Central Glass and Ceramic Research Institute \\ 196 Raja Subodh Chandra Mullick Road, \\ Calcutta 700 032, India
}

\begin{abstract}
A simple variational displacement phonon basis, obtained through the modified Lang-Firsov (MLF) transformation, is proposed to study the Holstein model. This phonon basis contains only one variational parameter, but capable of describing lattice distortions at distant sites from the charge carrier. Perturbation method based on this MLF basis is employed to calculate the singleelectron ground-state energy and the dispersion of the polaronic band. The ground-state $(k=0)$ energy obtained up to the second-order perturbation within this approach agrees well with the available numerical results for the entire range of coupling strength.
\end{abstract}

PACS numbers: $71.38 .+\mathrm{i}, 63.20 . \mathrm{kr}$

Keywords: Holstein model, D. electron-phonon interactions, Perturbation study

Self-localization of electrons in crystal lattices due to interaction with phonons constitutes one of the central problems in condensed matter physics. The simplest Holstein model [1], describing coupling of conduction electron with dispersionless phonons, has been studied in weak [2] as well as strong coupling [3] limits following either variational [4, 5, 6 or perturbation methods [7, 8, 9 . Numerical approaches including exact diagonalization (ED) techniques [7, 10], Quantum Monte Carlo calculations [11, Global-Local (GL) method [12], densitymatrix-renormalization-group (DMRG) technique 13 and recently developed ED technique with variational Hilbert space 14 are quite successful in evaluating the ground-state properties including various correlation functions in the entire range of electron-phonon (e-ph) coupling. The analytical methods, however, suffer from many limitations. Migdal approximation [2] is valid for a weakly-coupled adiabatic system while Lang-Firsov (LF) canonical transformation [3] is suitable only in the strongcoupling limit. No common consensus regarding proper choice of the phonon basis and applicability of perturbation theory has yet been reached for the whole range of $e$-ph coupling. So far the perturbative method has been employed using the LF basis only and no perturbation calculation based on the modified LF (MLF) basis is available. Recently, we [9] investigated the convergence of the perturbation expansion on a two-site single electron Holstein model using different phonon bases obtained through LF, MLF and MLF with squeezing (MLFS) transformations. It has been observed that for a wide region of e-ph coupling the perturbation corrections within the MLF method are much smaller and the convergence is much better compared to the LF approach.

Inspired with this result, we now attempt to apply the perturbation method based on a properly chosen MLF basis to the one-dimensional (infinite site) Holstein model and evaluate the perturbation corrections to the groundstate energy, wave function and the dispersion. The Hol- stein Hamiltonian for a single electron reads as

$$
\begin{aligned}
H & =\omega \sum_{i} b_{i}^{\dagger} b_{i}+\epsilon \sum_{i} n_{i}-t \sum_{i, \delta_{1}} c_{i}^{\dagger} c_{i+\delta_{1}} \\
& +\omega g \sum_{i}\left(b_{i}^{\dagger}+b_{i}\right) n_{i}
\end{aligned}
$$

where $i=1$ to $N$, denotes the site. $c_{i}\left(c_{i}^{\dagger}\right)$ is the annihilation (creation) operator for the electron at site $i$ and $n_{i}$ $\left(=c_{i}^{\dagger} c_{i}\right)$ is the corresponding number operator, $g$ denotes the on-site $e$-ph coupling strength and $t$ is the usual hopping integral. $b_{i}$ and $b_{i}^{\dagger}$ are the annihilation and creation operators, respectively, for the phonons corresponding to interatomic vibration at site $i$ and $\omega$ is the phonon frequency. $\delta_{1}$ runs over nearest-neighbor sites.

The spread and depth of the polaron may be studied conveniently within the framework of the MLF transformation where the lattice deformations produced by a charge carrier at different neighboring sites are treated as variational parameters [5, 6]. The MLF transformation on Eq. (1) gives $\tilde{H}=e^{R} H e^{-R}$

where

$$
\begin{aligned}
R & =\sum_{i} n_{i}\left[\lambda_{0}\left(b_{i}^{\dagger}-b_{i}\right)+\lambda_{1} \sum_{\delta_{1}}\left(b_{i+\delta_{1}}^{\dagger}-b_{i+\delta_{1}}\right)\right. \\
& \left.+\lambda_{2} \sum_{\delta_{2}}\left(b_{i+\delta_{2}}^{\dagger}-b_{i+\delta_{2}}\right)+\ldots+\lambda_{l} \sum_{\delta_{l}}\left(b_{i+\delta_{l}}^{\dagger}-b_{i+\delta_{l}}\right)+. .\right]
\end{aligned}
$$

and $\lambda_{0}, \lambda_{1}, \lambda_{2}, \ldots$ represent the variational lattice deformations around the charge carrier produced at the same, first-, second-, etc. neighbouring sites, respectively. $\delta_{l}$ runs over the $l$ th nearest neighbour sites. For LF transformation, $\lambda_{0}=g$ and $\lambda_{1}=\lambda_{2}=\ldots=\lambda_{l}=\ldots=0$.

The MLF transformed Hamiltonian is then obtained 
as

$$
\tilde{H}=\omega \sum_{i} b_{i}^{\dagger} b_{i}+\epsilon_{p} \sum_{i} n_{i}+H_{t}+H_{s}
$$

where

$$
\begin{aligned}
H_{t} & =-t \sum_{i, \delta_{1}} \exp \left(Y_{i}-Y_{i+\delta_{1}}\right) c_{i}^{\dagger} c_{i+\delta_{1}} \\
H_{s} & =\omega \sum_{i}\left(b_{i}^{\dagger}+b_{i}\right)\left[\left(g-\lambda_{0}\right) n_{i}-\lambda_{1} \sum_{\delta_{1}} n_{i+\delta_{1}}\right. \\
& \left.-\lambda_{2} \sum_{\delta_{2}} n_{i+\delta_{2}}-\lambda_{3} \sum_{\delta_{3}} n_{i+\delta_{3}}-. .\right] \\
Y_{i} & =\lambda_{0}\left(b_{i}^{\dagger}-b_{i}\right)+\lambda_{1} \sum_{\delta_{1}}\left(b_{i+\delta_{1}}^{\dagger}-b_{i+\delta_{1}}\right) \\
& +\lambda_{2} \sum_{\delta_{2}}\left(b_{i+\delta_{2}}^{\dagger}-b_{i+\delta_{2}}\right)+\lambda_{3} \sum_{\delta_{3}}\left(b_{i+\delta_{3}}^{\dagger}-b_{i+\delta_{3}}\right)+. .
\end{aligned}
$$

and

$$
\epsilon_{p}=\epsilon-\omega\left(2 g-\lambda_{0}\right) \lambda_{0}+2 \omega\left(\lambda_{1}^{2}+\lambda_{2}^{2}+\lambda_{3}^{2}+\ldots\right)
$$

is the polaron self-energy.

For the perturbation calculation following Marsiglio [7] we choose the basis set for the one-electron case as

$$
\left|\psi_{l}\left\{n_{i}\right\}\right\rangle=\frac{1}{\sqrt{N}} c_{l}^{\dagger}|0\rangle_{e}\left|n_{1} n_{2} \ldots n_{N}\right\rangle_{p h}
$$

which represents a state with $l$ th site occupied by the electron and $n_{1}, n_{2}, \ldots, n_{N}$ being the phonon numbers at sites $1,2, \ldots, \mathrm{N}$, respectively in the MLF displaced phonon basis. $l$ takes values from 1 to $N$ while $n_{1}, n_{2}$, ... from zero to infinity covering the whole Hilbert space. $H_{t}$ and $H_{s}$ are non-diagonal and the remaining part of the Hamiltonian is diagonal in this representation. The unperturbed energy of the state, described in Eq. (3), is given by $E_{\left\{n_{i}\right\}}^{(0)}=\epsilon_{p}+n_{T} \omega$ where $n_{T}\left(=\sum_{i} n_{i}\right)$ represents the total number of phonons in that state. The ground state corresponds to $n_{T}=0$ and has $N$-fold degeneracy since the electron can occupy any one of the $N$ sites. This degeneracy is lifted by considering the first-order energy correction which results in a polaronic band with energy $E_{k}=\epsilon_{p}-2 t_{e} \cos (k a)$ where $t_{e}=t \exp \left(-\left\{\left(\lambda_{0}-\lambda_{1}\right)^{2}+\right.\right.$ $\left.\left.\left(\lambda_{1}-\lambda_{2}\right)^{2}+\left(\lambda_{2}-\lambda_{3}\right)^{2}+..\right\}\right)$.

The eigenstates are now given by

$$
\left|\psi_{k}\right\rangle=\frac{1}{\sqrt{N}} \sum_{l} \exp \left(i \vec{k} \cdot \vec{R}_{l}\right) c_{l}^{\dagger}|0\rangle_{e}|0\rangle_{p h}
$$

and the unperturbed ground state $\left|\psi_{G}^{(0)}\right\rangle$ corresponds to $k=0$.

For perturbation calculation it is very tedious and inconvenient to deal with a phonon basis containing too many variational parameters. Previously we [9] observed that a choice of variational parameters which minimizes the ground-state energy, obtained within MLF and zerophonon averaging (ZPA) (i.e., from ${ }_{p h}\langle 0|\tilde{H}| 0\rangle_{p h}$ ) leads to a phonon basis where the convergence of the perturbation series is satisfactory. Here minimization of
$E_{k=0}=\epsilon_{p}-2 t_{e}$ (equivalent to the ground-state energy obtained by ZPA of $\tilde{H})$ with respect to parameters $\left(\lambda_{0}\right.$, $\left.\lambda_{1}, \ldots, \lambda_{l}\right)$ gives the general recursion relation between the variational parameters

$$
\frac{\lambda_{l-1}}{\lambda_{l}}=\frac{\lambda_{l-2}-2 \lambda_{l-1}+\lambda_{l}}{\lambda_{l-1}-2 \lambda_{l}+\lambda_{l+1}} .
$$

which is satisfied by a simple choice for the parameters

$$
\frac{\lambda_{1}}{\lambda_{0}}=\frac{\lambda_{2}}{\lambda_{1}}=\frac{\lambda_{3}}{\lambda_{2}}=\ldots=\frac{\lambda_{l}}{\lambda_{l-1}}=\ldots=r .
$$

A sum rule is also followed by $\lambda_{l}$ 's; for one-dimensional chain this is given by

$$
\lambda_{0}+2 \lambda_{1}+2 \lambda_{2}+\ldots+2 \lambda_{l}+\ldots=g .
$$

Eq. (7) is a consequence of the fact that the in-phase phonon mode (zero momentum) being a sum of lattice displacement operators at different sites $\left(\sum_{i}\left(b_{i}+b_{i}^{\dagger}\right)\right)$ couples only with the total number of electrons, which is a constant of motion. Eqs. (6) and (7) allow one to express all $\lambda_{l}$ 's in terms of a single variational parameter $r$. Thus, the obtained MLF phonon basis, though being capable of considering lattice distortions at all sites of the infinite chain, contains only one variational parameter.

The polaron self-energy may then be written in terms of $r$ as $\epsilon_{p}=\epsilon-\omega g^{2} \frac{(1-r)\left(1+4 r+r^{2}\right)}{(1+r)^{3}}$ and the effective hopping $t_{e}=t \exp \left(-g^{2} x^{3}\right)$ where $x=\frac{1-r}{1+r}$.

The expression for the off-diagonal matrix element between the ground state and an excited state is given by

$$
\begin{aligned}
H_{l\{n\}, G}^{\prime} & =\left\langle\psi_{l}\left\{n_{i}\right\}\left|\left(H_{t}+H_{s}\right)\right| \psi_{G}^{(0)}\right\rangle \\
& =-\frac{t_{e} y^{n_{T}}}{\sqrt{N}}\left[\frac{\left[(-1)^{n_{L}} r^{A}+(-1)^{n_{R}} r^{B}\right]}{\sqrt{\left(\ldots n_{l-1} ! n_{l} ! n_{l+1} ! \ldots\right)}}\right] \\
& +\frac{g \omega}{\sqrt{N}}\left[(1-x) \delta_{n_{l}, 1}-x\left[r\left(\delta_{n_{l-1}, 1}+\delta_{n_{l+1}, 1}\right)\right.\right. \\
& \left.\left.+r^{2}\left(\delta_{n_{l-2}, 1}+\delta_{n_{l+2}, 1}\right)+\ldots\right]\right] \delta_{n_{T}, 1}
\end{aligned}
$$

$$
\text { where } \begin{aligned}
y & =g x(1-r), \\
n_{L} & =n_{l-1}+n_{l-2}+n_{l-3}+\ldots, \\
n_{R} & =n_{l+1}+n_{l+2}+n_{l+3}+\ldots, \\
A & =n_{l+1}+n_{l-2}+2\left(n_{l+2}+n_{l-3}\right) \\
& +3\left(n_{l+3}+n_{l-4}\right)+. ., \\
B & =n_{l-1}+n_{l+2}+2\left(n_{l-2}+n_{l+3}\right) \\
& +3\left(n_{l-3}+n_{l+4}\right)+\ldots .
\end{aligned}
$$

The second-order correction to the ground-state energy is given by

$$
\begin{aligned}
E_{0}^{(2)} & =-\frac{2 g^{2}}{\omega}\left[\omega r-t_{e}(1-r)^{2}\right]^{2} \frac{(3+r)}{(1+r)^{3}} \\
& -\frac{1}{N} \sum_{l} \sum_{n_{T} \geq 2} \frac{t_{e}^{2} y^{2 n_{T}}}{\left(n_{T} \omega\right)} \frac{\left[(-1)^{n_{L}} r^{A}+(-1)^{n_{R}} r^{B}\right]^{2}}{\left(\ldots n_{l-1} ! n_{l} ! n_{l+1} ! \ldots\right)}
\end{aligned}
$$


and the first-order correction to the ground-state wave function is obtained as,

$$
\begin{array}{r}
\left|\psi_{G}^{(1)}\right\rangle=-\frac{1}{\omega \sqrt{N}} \sum_{l=1}^{N} c_{l}^{\dagger}|0\rangle_{e}\left[\left\{\omega r-t_{e}(1-r)^{2}\right\}\right. \\
\left.\sum_{m}\left[\frac{2 g}{1+r} \delta_{m, l}-\sum_{p} g x r^{p-1} \delta_{m, l+p}\right]\left|00 . .01_{m} 0 . .\right\rangle_{p h}\right] \\
-\frac{1}{\omega \sqrt{N}} \sum_{l=1}^{N} c_{l}^{\dagger}|0\rangle_{e} \sum_{n_{T} \geq 2} \frac{H_{l\{n\}, G}^{\prime}}{\left(n_{T}\right)}\left|n_{1} n_{2} \ldots n_{N}\right\rangle_{p h}
\end{array}
$$

Now one has to make a proper choice of $r$. In addition to Eq. (6) the minimization of $E_{k=0}$ yields a condition $\omega r=t_{e}(1-r)^{2}$, which gives

$$
r=\frac{\left(2 t_{e}+\omega\right)-\sqrt{4 t_{e} \omega+\omega^{2}}}{2 t_{e}}
$$

It is interesting to note that for this choice of $r$ the offdiagonal matrix element between the ground state and any excited state with a single phonon $\left(n_{T}=1\right)$ becomes zero and this is consistent with our previous studies [9]. Correspondingly, the first term in Eq. (10) as well as in Eq. (11) vanishes.

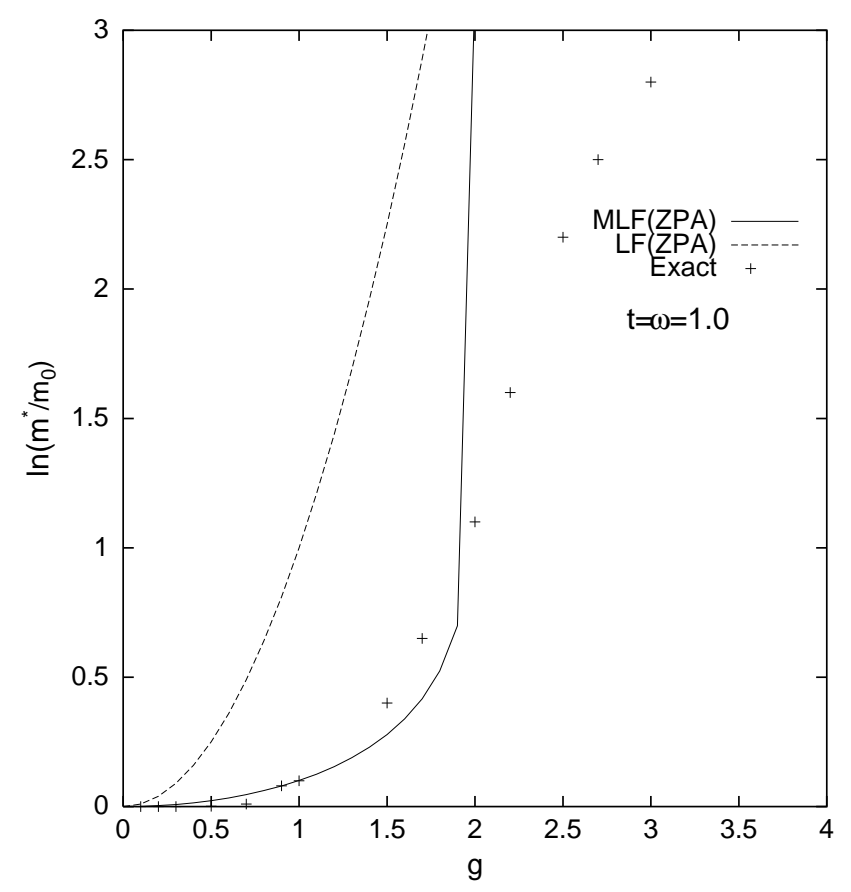

FIG. 1: Plot of logarithm of the effective mass $m^{*} / m_{0}$ as function of $g$ for $t=\omega=1.0$ within MLF and LF approach using the unperturbed ground-state wave function. '+' shows the results of Ref [14].

For real materials of interest the Hamiltonian may involve other interactions in addition to the Holstein-type $e$-ph interaction and the perturbation calculations for such complicated systems may not be possible. The common practice in such cases is to make LF or MLF transformation followed by ZPA. It may be mentioned that ZPA is equivalent to the zeroth (first) order of perturbation as far as the wave function (energy) is concerned. The success of the ZPA depends on the choice of the phonon basis. One of the advantages of the MLF phonon basis over LF basis is that the second-order perturbation correction $\left(E_{0}^{(2)}\right)$ within MLF basis is much smaller than that within LF basis except in the strong-coupling regime where both the bases become equivalent. For instance, for $\omega=t=1, E_{0}^{(2)}(M L F)=-0.01746$ and -0.1849 while $E_{0}^{(2)}(L F)=-1.3538$ and -0.4801 for $g=1.0$ and 1.7 , respectively.

A comparison of the effective mass of the polaron obtained within the MLF and LF with ZPA may be helpful. We have calculated the effective mass of the polaron using the standard formula $\frac{m_{0}}{m^{*}}=\left.\frac{1}{2 t} \frac{\delta^{2} E(k)}{\delta k^{2}}\right|_{k=0}$ (where $\mathrm{m}_{0}=\frac{1}{2 t}$ ) within the ZPA of LF and MLF Hamiltonian. These results along with the exact numerical result of Ref. 14 are presented in Fig. 1. The proximity of the MLF results with the exact one indicates that the MLF basis is a better choice.

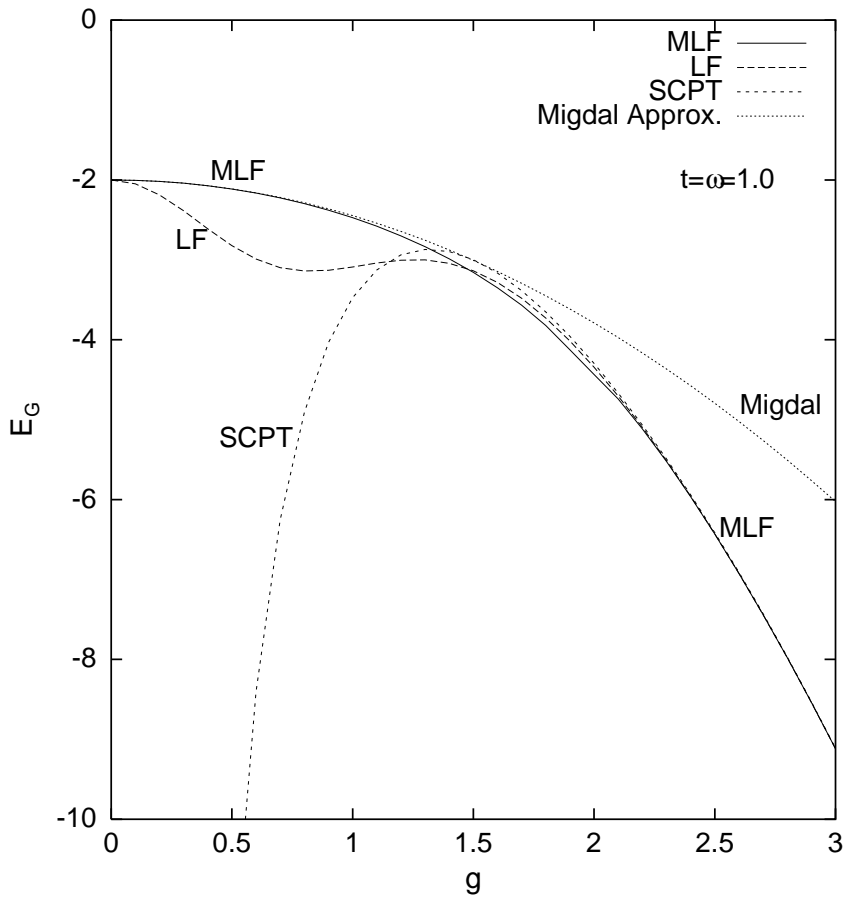

FIG. 2: Plot of the ground-state energy (in unit of $t$ ) obtained by considering perturbation corrections up to secondorder within MLF, LF method and also within the weakcoupling (self-consistent Migdal approximation) and strongcoupling perturbation expansion (SCPT) obtained from Ref. [7] as a function of $g$ for $t=\omega=1.0$. 
In Fig. 2 we have shown the ground-state energy calculated up to the second-order perturbation correction within the LF (corresponds to $r=0$ ) and the MLF phonon basis. Energies calculated from the weak coupling (self-consistent Migdal approximation) and strong coupling perturbation limit, obtained from (Eqs. (7) and (C.17) of) Ref. 7], are also plotted. From comparison with the GL results [12] we find that the MLF energy almost coincides with the exact energy in the entire regime of $g$. The weak-coupling method fails for large $g$ whereas the strong-coupling perturbation expansion of Ref. [7] breaks down and the LF perturbation result deviates much from the exact energy for low $g(<1.4)$.

To find out the energy dispersion within the MLF method the energy $E_{k}$ has been calculated up to the second-order perturbation. The second-order perturbation correction to $E_{k}$ within the MLF approach is obtained as

$$
\begin{aligned}
E_{k}^{(2)} & =-\frac{2 g^{2}}{\omega}\left[\frac{(3+r)}{(1+r)^{3}}\left(\omega r-t_{e}(1-r)^{2} \cos (k a)\right)^{2}\right. \\
& \left.+\frac{(1-r)^{3}}{(1+r)} t_{e}^{2} \sin ^{2}(k a)\right] \\
& -\frac{1}{N} \sum_{l} \sum_{n_{T} \geq 2} \frac{t_{e}^{2} y^{2 n_{T}}}{\left(n_{T} \omega\right)} \frac{\left(X^{2}+Y^{2}+2 X Y \cos (2 k a)\right)}{\left(\ldots n_{l-1} ! n_{l} ! n_{l+1} ! \ldots\right)}
\end{aligned}
$$

where $X=(-1)^{n_{L}} r^{A}$ and $Y=(-1)^{n_{R}} r^{B}, n_{L}, n_{R}$, and $A$ and $B$ are defined in Eq. (9).

The corresponding expression for $E_{k}^{(2)}$ within the LF approach is

$$
\begin{aligned}
E_{k}^{(2)} & =-\frac{2 t_{e}^{2}}{\omega}\left[\sum_{n=1}^{\infty} \frac{2}{n} \frac{g^{2 n}}{n !} \cos ^{2}(k a)\right. \\
& \left.+\sum_{n=0}^{\infty} \sum_{m=1}^{\infty} \frac{g^{2(n+m)}}{(n+m) n ! m !}\right]
\end{aligned}
$$

where $t_{e}=t e^{-g^{2}}$.

We have calculated $E_{k}^{(2)}$, hence $E_{k}$ up to the secondorder perturbation within the LF and MLF approach. The second-order correction $E_{k}^{(2)}$ and the ratio $E_{k}^{(2)} / E_{k}$ for different $k$ values are listed in Table I for several values of $g$ for both the MLF and the LF approach for $t=\omega=1$.

The following features are evident from our study: (i) The second-order MLF corrections are appreciably smaller than the corresponding LF corrections for low values of $g$ and $k$. (ii) For small and intermediate values of $g$ (e.g. $g=0.5$ and 1.0) the MLF correction increases with increasing $k$, however the MLF corrections are less than the corresponding LF values in the entire region of $k$ except at $k a=\pi / 2$ where both the MLF and LF corrections are same. In fact, for $k a=\pi / 2$ the value of $r$ becomes zero within the present MLF approach so the MLF basis reduces to the LF basis for $k a=\pi / 2$.
TABLE I: $k a / \pi$, second-order correction energy $\left(E_{k}^{(2)}\right.$, in unit of $t$ ) and realtive second-order correction $\left(E_{k}^{(2)} / E_{k}\right)$ for MLF and LF method respectively, (for $g=0.5,1$ and 2.0, $t=\omega=$ 1.0) for the ground state of many-site one polaron problem.

\begin{tabular}{ccccc}
\hline \multicolumn{4}{c}{ MLF } & \multicolumn{2}{c}{$\mathrm{LF}$} \\
$k a / \pi$ & $E_{k}^{(2)}$ & $E_{k}^{(2)} / E_{k}$ & $E_{k}^{(2)}$ & $E_{k}^{(2)} / E_{k}$ \\
\hline \multicolumn{1}{c}{$=0.5$} \\
\hline 0.00 & -0.0010180 & 0.000482 & -1.014952 & 0.359587 \\
0.10 & -0.0094835 & 0.004680 & -0.953203 & 0.355067 \\
0.20 & -0.0364071 & 0.020493 & -0.791541 & 0.343899 \\
0.30 & -0.0881567 & 0.062911 & -0.591716 & 0.336728 \\
0.40 & -0.1841571 & 0.189624 & -0.430054 & 0.370296 \\
0.50 & -0.3683048 & 0.595669 & -0.368305 & 0.595669 \\
\hline
\end{tabular}

\begin{tabular}{crrrr}
$\mathrm{g}=1.0$ & & & & \\
\cline { 1 - 4 } 0.00 & -0.0174239 & 0.007045 & -1.353833 & 0.438192 \\
0.10 & -0.0501885 & 0.020757 & -1.285706 & 0.430657 \\
0.20 & -0.1526451 & 0.067293 & -1.107347 & 0.409736 \\
0.30 & -0.3373983 & 0.162439 & -0.886884 & 0.382384 \\
0.40 & -0.5934159 & 0.310214 & -0.708525 & 0.365995 \\
0.50 & -0.6403983 & 0.390392 & -0.640398 & 0.390392 \\
\hline
\end{tabular}

\begin{tabular}{crrrr}
$\mathrm{g}=2.0$ & & & & \\
\cline { 1 - 4 } 0.00 & -0.3833072 & 0.086432 & -0.305533 & 0.070364 \\
0.10 & -0.3700764 & 0.083773 & -0.303269 & 0.069908 \\
0.20 & -0.3426137 & 0.078217 & -0.297343 & 0.068718 \\
0.30 & -0.3159575 & 0.072782 & -0.290017 & 0.067265 \\
0.40 & -0.3020421 & 0.070014 & -0.284090 & 0.066138 \\
0.50 & -0.2814511 & 0.065737 & -0.281826 & 0.065819 \\
\hline
\end{tabular}

(iii) The second-order perturbation correction within the LF method is symmetric around $k a=\pi / 2$ as evident from Eq. (13). It is also minimum for $k a=\pi / 2$. (iv) For small and intermediate values of $g$ the second-order LF corrections are quite high. As a result $E_{k}$ up to the second-order correction within the LF may not be an accurate estimate of $E_{k}$. Nevertheless we have cited the LF values for comparison with the MLF values. (v) In a range $1.9<g<2.2$ (for $t=\omega=1$ ) the LF second-order corrections are slightly less than the corresponding MLF corrections. For higher values of $g$ the MLF phonon basis reduces to the LF phonon basis and the results by these two methods become equivalent. (vi) With increasing $g$ the second-order LF correction decreases considerably and becomes more and more $k$-independent. $E_{k}^{(2)} / E_{k}$ within LF is around 0.027 for $g=2.5$, for the whole region of $k$.

In Fig. $3 a$ we plot $E_{k}$ vs. $k a / \pi$ for $g=0.5$ and 1.0 for the MLF approach and in Fig. $3 \mathrm{~b}$ a comparison with LF result and the numerically exact results [12, 13, 14, is shown for $g=1$. The MLF result is close to the numerically exact results only for low $k$ but deviates considerably from the exact curve for higher values of $k$. In this region the second-order corrections are increasingly higher implying that higher-order corrections are to be included to obtain accurate results. The LF re- 

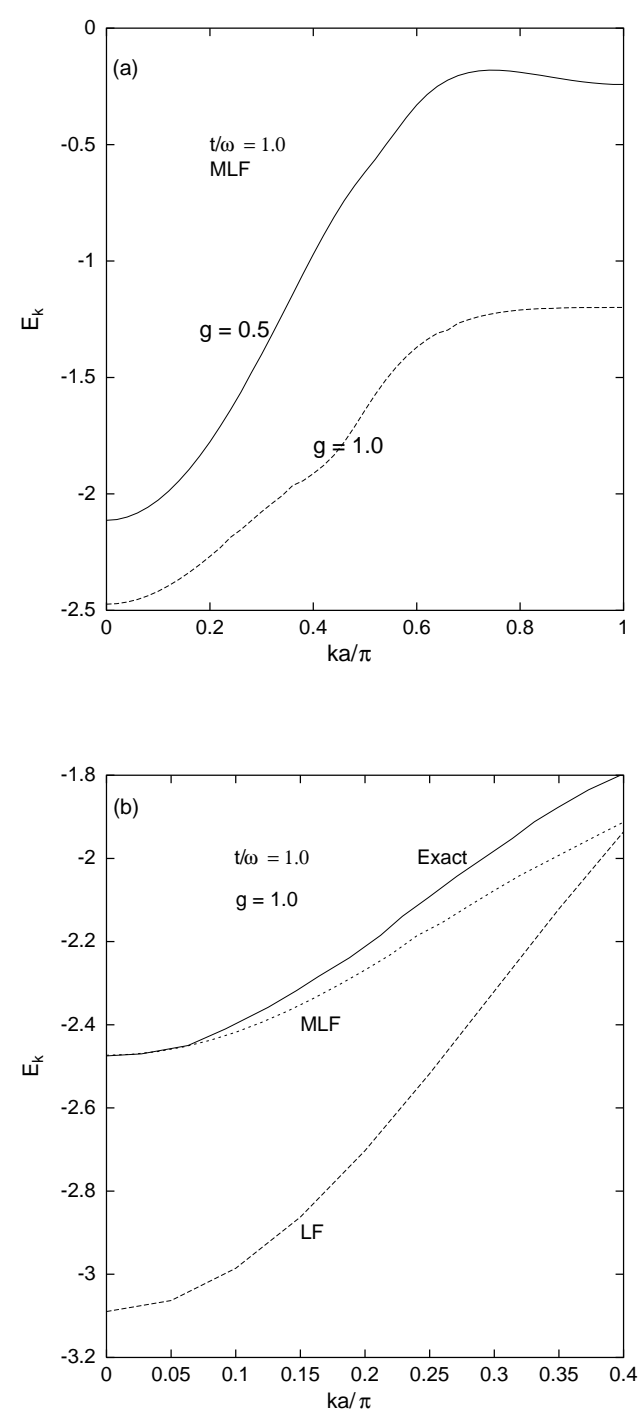

FIG. 3: Plot of $E_{k}$ (in unit of $t$ ) vs $k a / \pi$ for $t=\omega=1.0$ : (a) Within the MLF method for $g=0.5$ and 1.0. (b) Within the MLF and LF methods for $g=1.0$; the solid line shows the exact results from Ref.[14]. sult is much less satisfactory than the MLF result for $g=1$. The MLF perturbation method predicts a flat band (FIG. 3(b)) in the region $0.7 \pi \leq k a \leq \pi$ which is consistent qualitatively with the exact result [12, 14] for $g=1.0$. However, the values of $E_{k}$ predicted by the MLF in this region is found to be not consistent with the exact results.

For large $g$ the LF second-order corrections are small in the entire region of $k$, hence the dispersion given by the LF up to the second-order should be satisfactory. However, no numerical result for $E_{k}$ for large $g$ is available to us for a comparison.

In summary, in this paper we have proposed a simple MLF phonon basis containing a single variational parameter but capable of describing lattice distortions at distant sites from the charge carrier. For low and intermediate values of $g$ the second-order perturbation correction to the energy within this approach is much smaller than that corresponding to the LF approach. It appears that the ZPA with this MLF basis gives much more accurate results than that of ZPA with the LF basis. One representative calculation of effective mass is presented which corroborates this feature. The groundstate $(k=0)$ energy, calculated up to the second-order within the MLF approach agrees well with exact results for the entire range of coupling strength. For $g \leq 1.0$, the second-order correction within the MLF is small for low $k$ values and in this region the values of $E_{k}$ (band dispersion) predicted by the MLF is consistent with the numerically exact result. For large $g$ the LF perturbation corrections are small and the band dispersion predicted by the LF method is expected to be satisfactory. Computation of higher-order corrections would shed light on this issue.

Electronic address for correspondence: moon@cmp.saha.ernet.in
[1] T. Holstein, Ann. Phys. (NY) 8, (1959) 325.

[2] A. B. Migdal, Sov. Phys. JETP 7, (1958) 996.

[3] L. G. Lang and Yu A. Firsov, Sov. Phys. JETP 16, (1963) 1301.

[4] Y. Toyozawa, Prog. Theor. Phys. 26, (1961) 29.

[5] D. Feinberg, S. Ciuchi and F. de Pasquale, Int. J. Mod. Phys B 4, (1990) 1317.

[6] A. N. Das and S. Sil, Phyica C 207, (1993) 51; J. Phys.: Condens. Matter 5, (1993) 1; P. Choudhury and A. N. Das, Int. J. Mod. Phys B 15, (2001) 1923.

[7] F. Marsiglio, Physica C 244, (1995) 21.

[8] Marco Zoli, Phys. Rev. B 61, (2000) 13.

[9] A. N. Das and Jayita Chatterjee, Int. J. Mod. Phys B 13, (1999) 3903; Jayita Chaterjee and A. N. Das, Phys. Rev. B 61, (2000) 4592.
[10] G. Wellein and H. Fehske, Phys. Rev. B 58, (1998) 6208; E. V. L de Mello and J. Ranninger, ibid 55 (1997) 14872.

[11] P. E. Kornilovitch and E. R. Pike, Phys. Rev. B 55, (1997) R8634.

[12] A. H. Romero et al, Phys. Rev. B 59, (1999) 13.

[13] E. Jeckelmann and S. R. White, Phys. Rev. B 57 (1998) 6376.

[14] J. Bonca, S. A. Trugman and I. Batistic, Phys. Rev. B 60 (1999) 1633; Li-Chung Ku, S. A. trugman and J. Bonca, cond-mat/0109282 (2001).

This work is partly supported by the Project No. SP/S2/M-62/96, sponsered by Department of Science and Technology, India. 